\title{
Safety, efficacy, and immunogenicity of an inactivated influenza vaccine in healthy adults: a randomized, placebo-controlled trial over two influenza seasons
}

\author{
Lisa A Jackson*1, Manjusha J Gaglani2 ${ }^{2}$, Harry L Keyserling³ ${ }^{3}$ John Balser ${ }^{4}$, Nancy Bouveret ${ }^{5,6}$, Louis Fries ${ }^{7}$ and \\ John J Treanor8
}

\begin{abstract}
Background: Seasonal influenza imposes a substantial personal morbidity and societal cost burden. Vaccination is the major strategy for influenza prevention; however, because antigenically drifted influenza A and B viruses circulate annually, influenza vaccines must be updated to provide protection against the predicted prevalent strains for the next influenza season. The aim of this study was to assess the efficacy, safety, reactogenicity, and immunogenicity of a trivalent inactivated split virion influenza vaccine (TIV) in healthy adults over two influenza seasons in the US.

Methods: The primary endpoint of this double-blind, randomized study was the average efficacy of TIV versus placebo for the prevention of vaccine-matched, culture-confirmed influenza (VMCCI) across the 2005-2006 and 2006-2007 influenza seasons. Secondary endpoints included the prevention of laboratory-confirmed (defined by culture and/or serology) influenza, as well as safety, reactogenicity, immunogenicity, and consistency between three consecutive vaccine lots. Participants were assessed actively during both influenza seasons, and nasopharyngeal swabs were collected for viral culture from individuals with influenza-like illness. Blood specimens were obtained for serology one month after vaccination and at the end of each influenza season's surveillance period.

Results: Although the point estimate for efficacy in the prevention of all laboratory-confirmed influenza was 63.2\% ( $97.5 \%$ confidence interval [CI] lower bound of $48.2 \%$ ), the point estimate for the primary endpoint, efficacy of TIV against VMCCl across both influenza seasons, was $46.3 \%$ with a $97.5 \% \mathrm{Cl}$ lower bound of $9.8 \%$. This did not satisfy the pre-specified success criterion of a one-sided $97.5 \% \mathrm{Cl}$ lower bound of $>35 \%$ for vaccine efficacy. The VMCCl attack rates were very low overall at $0.6 \%$ and $1.2 \%$ in the TIV and placebo groups, respectively. Apart from a mismatch for influenza B virus lineage in 2005-2006, there was a good match between TIV and the circulating strains. TIV was highly immunogenic, and immune responses were consistent between three different TIV lots. The most common reactogenicity events and spontaneous adverse events were associated with the injection site, and were mild in severity.

Conclusions: Despite a good immune response, and an average efficacy over two influenza seasons against laboratory-confirmed influenza of $63.2 \%$, the pre-specified target (lower one-sided $97.5 \%$ confidence bound for efficacy $>35 \%$ ) for the primary efficacy endpoint, the prevention of $\mathrm{VMCCl}$, was not met. However, the results should be interpreted with caution in view of the very low attack rates we observed at the study sites in the 2005-2006 and 2006-2007, which corresponded to relatively mild influenza seasons in the US. Overall, the results showed that TIV has an acceptable safety profile and offered clinical benefit that exceeded risk.
\end{abstract}

Trial registration: NCT00216242

(c) 2010 Jackson et al; licensee BioMed Central Ltd. This is an Open Access article distributed under the terms of the Creative Commons :H Wed Central Attribution License (http://creativecommons.org/licenses/by/2.0), which permits unrestricted use, distribution, and reproduction in any medium, provided the original work is properly cited. 


\section{Background}

Annual epidemics of influenza due to influenza A and B viruses remain a substantial cause of morbidity and mortality worldwide, particularly among vulnerable groups such as people aged $\geq 65$ years, children aged $<2$ years, and people with chronic medical conditions [1-3]. In addition to these identified risk groups, influenza is responsible for a substantial burden of illness, absenteeism, and resultant societal costs among otherwise healthy working adults [4-7]. Trivalent inactivated influenza vaccines (TIV) containing antigens of two influenza A strains (one $\mathrm{A} / \mathrm{H} 1 \mathrm{~N} 1$ and one $\mathrm{A} / \mathrm{H} 3 \mathrm{~N} 2$ ), in combination with antigens of one influenza $B$ strain, provide the current standard for influenza prevention. Because one or more new antigenically drifted variants circulate annually, the vaccines must be updated to provide optimal protection against the predicted prevalent strains for the next influenza season [8-10]. The World Health Organization (WHO), as well as the US Food and Drug Administration (FDA) Center for Biologics Evaluation and Research, provide annual guidance for strain selection based on new drift variants detected through a global influenza surveillance network $[8,9]$.

Clinical trial data have repeatedly shown that TIVs can be protective against seasonal influenza, including seasons when the surface antigens of the prevalent virus(es) match the vaccine strains, and some seasons when a drifted strain circulates, although efficacy can be reduced as a result of substantial antigenic drift [8,11-14]. However, many TIV studies have been either too small to provide narrow confidence limits about the point estimates for efficacy, or have used serological criteria to define the influenza infection endpoint, which unlike virus detection by culture or molecular methods, may bias the results in favor of the vaccine because detection of infection by seroconversion may be adversely affected by prior elevation of baseline titers in vaccinated, but not unvaccinated subjects $[15,16]$. Furthermore, marked variations in efficacy estimates have been observed from season to season, even when the same investigators have applied identical methods to evaluate the same type of vaccine formulation in consecutive years $[12,13]$.

In this paper, we describe an efficacy, safety, and immunogenicity study of a trivalent inactivated split virus influenza vaccine (TIV; marketed, depending on the countries, as Fluviral', FluLaval ${ }^{\mathrm{m}}$, or Griplaval ${ }^{\mathrm{mm}}$ trademarks of the GlaxoSmithKline group of companies). The product, first licensed in Canada in 1992, has undergone several process refinements, including introduction of a concentration step, homogenization, and sterile filtration. Following clinical studies of safety and immunogenicity

\footnotetext{
* Correspondence: jackson.l@ghc.org

${ }^{1}$ Group Health Research Institute, Seattle, Washington, USA
}

in adults conducted in Canada and the US in the 20042005 influenza season, the product received accelerated approval from the FDA in 2006 [17]. The randomized, placebo-controlled study reported in this paper is part of the post-approval clinical trial program, conducted to confirm manufacturing consistency, and to further assess the clinical benefits of this TIV formulation using culture-confirmed efficacy endpoints[17].

The study included healthy adults aged 18 to 49 years in the US, and in order that a placebo control could be ethically employed, high-risk patients for whom annual influenza vaccine is recommended by the Advisory Committee on Immunization Practices (ACIP) were not eligible for inclusion [18]. The primary clinical endpoint was based upon rates of culture-confirmed influenza illness caused by A and/or B virus strains antigenically matching those in the vaccine. The study was conducted over the 2005-2006 and 2006-2007 influenza seasons.

\section{Methods \\ Design and participants}

This was a randomized, double-blind, placebo-controlled study of the efficacy of TIV in prevention of vaccinematched, culture-confirmed influenza (VMCCI) conducted in the 2005-2006 and 2006-2007 influenza seasons in the US.

The original primary outcome measure defined by the study protocol was the average vaccine efficacy over two consecutive seasons in the prevention of culture-confirmed influenza. In correspondence following the 20052006 season, the FDA Center for Biologics Evaluation and Research noted that the season was marked by a significant frequency of circulation of influenza virus strains that were antigenically-drifted from those in the vaccine, and required that the protocol be modified to assess the average efficacy against VMCCI across both seasons as the primary measure of vaccine efficacy.

Male and female volunteers aged 18 to 49 years inclusive were eligible to participate if they were clinically healthy, understood the study procedures, had access to telephone contact throughout study, and provided informed written consent. In Season 1, eligible participants were enrolled at 37 centers, and in Season 2, eligible participants were enrolled at 44 centers.

Exclusion criteria included: a significant acute or chronic, or medical or psychiatric illness requiring institution of new medical or surgical treatment, or a significant dose alteration for uncontrolled symptoms or drug toxicity within 3 months; diagnosis of cancer, or treatment for cancer, within 3 years; systolic blood pressure $\geq$ $140 \mathrm{mmHg}$, diastolic blood pressure $\geq 90 \mathrm{mmHg}$; a health condition placing the potential subject in a risk group recommended for routine influenza immunization by Advisory Committee on Immunization Practices (ACIP), 
e.g. chronic pulmonary (including asthma), cardiovascular, renal, hepatic, hematological, or metabolic disorders (including diabetes mellitus); immunosuppressive illness, recent/ongoing receipt of immunosuppressive therapy, or human immunodeficiency virus infection [18]; recent administration of other vaccine or immunoglobulin, contraindication to influenza vaccine, or previous influenza vaccination within 9 months. A negative pregnancy urine test at screening was required for women of childbearing potential before inclusion into the study, and women were required to use reliable contraception throughout the study. Individuals employed in professions prone to influenza transmission to or from high-risk populations, and individuals living in the same household as an immunocompromised person were not eligible to participate (also based on ACIP recommendations [18]).

In order to allow an independent assessment of efficacy in each season, participants who were enrolled in the study during 2005-2006 (Season 1) were not eligible for enrollment during 2006-2007 (Season 2).

The study was conducted in accordance with Good Clinical Practice, US regulatory requirements, and the Declaration of Helsinki. This protocol and consent for this multi-centered study were approved by six (6) independent ethics committees/institutional review boards as required to provide ethical oversight for 44 sites. The names of these bodies are on file with the corresponding author.

\section{Vaccine allocation and administration}

Treatment allocation was determined by blocked, stratified randomization with a 1:1 distribution to TIV or placebo; randomization was stratified by study center, age (18-34 and 35-49 years), and the subject's report of previous recent receipt (within $\leq 2$ years) of TIV. Each study center had a pre-determined sequence of randomization numbers which were allocated sequentially to eligible subjects. Participants were allocated equally among 3 different vaccine lots. Clinic staff (excluding the nurse giving the vaccine), were blinded to the treatment group until the study was complete.

Participants received a single injection of TIV (FluLa$\mathrm{val}^{\mathrm{m}}$, a trademark of the GlaxoSmithKline group of companies; manufactured by ID Biomedical Corporation of Quebec [IBD-Q], Canada), or saline placebo injection. Each $0.5 \mathrm{~mL}$ dose of TIV contained $15 \mu \mathrm{g}$ of hemagglutinin (HA) antigen of each recommended influenza strain. Antigens for Season 1 (2005-2006) were A/New Caledonia/20/1999 (H1N1), A/New York/55/2004 (H3N2, A/ California/7/2004-like), and B/Jiangsu/10/2003 (B/ Shanghai/361/2002-like). Antigens for Season 2 (20062007) were $\mathrm{A} / \mathrm{New}$ Caledonia/20/1999 (H1N1) virus, A/ Wisconsin/67/2005 (H3N2), and B/Malaysia/2506/2004. Three consecutive vaccine lots were used in each season.

\section{Outcomes}

The primary endpoint was the average efficacy over Seasons 1 and 2 for the prevention of culture-confirmed influenza illness due to influenza A or B virus strains antigenically matching those in the vaccine (i.e. vaccinematched, culture-confirmed influenza; VMCCI). The case definition of VMCCI required the presence of influenza-like illness (ILI), defined as symptoms that interfered with normal daily activities and that included cough, and at least 1 additional symptom from among fever (oral temperature $>37.7^{\circ} \mathrm{C} / 99.9^{\circ} \mathrm{F}$ ), headache, myalgia and/or arthralgia, chills, rhinorrhea/nasal congestion, and sore throat. Participants meeting the definition for ILI and with concurrent isolation from a nasopharyngeal swab of an influenza A and/or B virus isolate antigenically matching a vaccine strain for the relevant year were considered to be cases of VMCCI.

Co-primary safety endpoints were the assessment of solicited local and general reactogenicity events occurring between 0 and 3 days of vaccination, and unsolicited spontaneous adverse events (AEs) for a minimum of 135 days post-vaccination.

The average efficacy over Seasons 1 and 2 against culture-confirmed influenza illness $(\mathrm{CCI})$, or laboratoryconfirmed influenza illness (LCI) were secondary endpoints. CCI was defined as ILI with any influenza A or B virus isolate by culture, and LCI was defined as one or both of CCI or ILI with a 4-fold increase in hemagglutination-inhibiting (HI) serum antibody titers to a circulating influenza virus strain between Day 21 ( \pm 4 days) postvaccination and Final Visit specimens obtained after the end of the influenza season. The average efficacy across both seasons was also assessed according to age, prior vaccination status, and gender; but no attempt was made to provide statistical power for hypothesis tests within these subgroups.

Immunogenicity endpoints were $\mathrm{HI}$ assay-based Day 21 seroconversion and seroprotection rates, geometric mean fold-rises (GMFR) between Day 0 and Day 21, and lot equivalence according to reciprocal GMTs at Day 21 in Season 1.

\section{Surveillance}

The surveillance period in each season was $~ 15$ weeks. Surveillance for influenza was conducted between 14 November 2005 through 30 April 2006 (Season 1), and 13 November 2006 through 30 April 2007 (Season 2). Throughout the influenza season, participants were instructed to report symptoms meeting the definition of ILI via a study-specific, toll-free telephone number within 48 hours of symptom onset, and to record temperatures and symptoms during ILI episodes. This passive surveillance method was accompanied by active surveillance in which ILI symptoms were solicited during 
weekly outbound telephone contact. Local visiting nurses were dispatched to participants fulfilling the definition of ILI within 24 hours of reporting, and obtained nasopharyngeal and oropharyngeal swabs for viral culture. To ensure that influenza illnesses were detected, participants could be swabbed more than once.

\section{Antigenic characterization and serology}

Blood samples were taken before vaccination on Day 0 , and at the next study visit on Day 21 ( \pm 4 days), and at the Final Visit, which was within 4 weeks following the end of the surveillance period. Following serum separation, samples were frozen until analysis. Combined nasopharyngeal and oropharyngeal swab samples from participants reporting ILI were refrigerated and transported to a central laboratory (Covance Central Laboratory Services, Inc., Indianapolis, US) for influenza virus culture and assessment of CCI. Samples were cultured using primary monkey kidney cells, and influenza A and B viruses were identified using immunofluorescent staining with influenza-specific monoclonal antibodies. Influenza A viruses were further sub-typed by immunofluorescence using $\mathrm{H} 1$ and $\mathrm{H} 3$ specific reagents.

Detailed antigenic characterization and vaccine match analysis (i.e. VMCCI) of the influenza viral isolates was determined by serologic methods in the laboratory of Dr John Treanor (University of Rochester Medical Center [URMC], New York, US), using ferret sera for influenza A virus strains (kindly provided by Dr Alexander Klimov, CDC, Atlanta, Georgia, US), or WHO typing sera for B lineages. Isolates were considered to be 'vaccine matching' if there was a $\leq 4$-fold difference in the HI titer of the subject isolate strain and the vaccine prototype strain with reference anti-serum [19]. Testing included TIV virus strains, and drifted influenza $\mathrm{A}$ virus strains or alternate-lineage influenza B virus strains circulating in each year. The isolate type and subtype were also confirmed using reverse transcription polymerase chain reaction (RT-PCR; GSK Biologicals, Rixensart, Belgium). RT-PCR confirmed the isolate type and subtype determined by immunofluorescence and/or HI testing for $100 \%$ of isolates.

To evaluate the endpoint of LCI, Day 21 and Final Visit sera of all participants who reported ILI, irrespective of culture-confirmation, were tested for seroconversion. Immunogenicity of the vaccine was examined by testing of Day 0 and Day 21 serum samples in a randomly selected subset of participants. Immunogenicity was assessed using established HI assay methods performed at the IBD-Q analytic laboratory (IBD-Q/GlaxoSmithKline Biologicals North America Clinical Immunology Laboratory, Laval, Quebec, Canada) [20].

\section{Reactogenicity and safety}

After the injection, participants were observed for 30 minutes for local and/or systemic reactogenicity, and oral temperature was taken. Participants received memory aids to record the severity of injection site reactions, reactogenicity events, and general solicited adverse events (AEs). Definitions of events and severity grades were provided with the memory aids. Participants reported immediate reactogenicity data (within 30 minutes of dosing) before leaving the clinic, and solicited reactogenicity symptoms (occurring on Day 0 from 30 minutes postvaccination to the evening of Day 3) no later than 8 days post-vaccination via an Interactive Voice Response System (IVRS).

Data on AEs and serious adverse events (SAEs) were reported throughout the entire study period. AEs were captured using electronic case report forms and coded by preferred term and primary system organ class (SOC) using the Medical Dictionary for Regulatory Activities (MedDRA).

\section{Sample size}

Sample size calculations were based on the FarringtonManning method, as implemented in the PASS software package. We assumed a vaccine efficacy of $70 \%$ and a rate of culture-confirmed influenza (CCI) of $2.0 \%$ in placebo recipients in each season. The originally planned sample size was selected to provide $80 \%$ power in a one-sided test with type 1 error $=0.025$ to reject the null hypothesis that the average vaccine efficacy was less than $35 \%$. An adjustment of $10 \%$ was applied to account for potential dropouts, resulting in a target of 3187 participants per study arm across both seasons (total 6374). After the end of Season 1, at the request of the regulatory authority (US FDA, Center for Biologics Evaluation and Research), the primary study endpoint was changed from CCI to vaccine-matching, culture-confirmed influenza illness (VMCCI).

As specified in the protocol, at the end of Season 1 an unblinded, independent statistician, who was not otherwise involved in the analysis, evaluated the actual rate of CCI detected during Season 1. This had originally been planned to ensure that the estimated sample size across both seasons would confer sufficient power to demonstrate average efficacy against CCI with the requisite degree of precision and to allow adjustment of the sample size in Season 2 if needed. Given the change in the primary endpoint, this determination also involved estimation of the proportion of influenza isolates identified from the placebo group that would be antigenically matched to the vaccine strains, to ensure that the study would be adequately powered for the endpoint of VMCCI. Therefore, the statistician assumed that $66.8 \%$ of influenza isolates identified in the placebo group in Sea- 
son 1 (all of which had not yet been sub-typed) were antigenically matched to the vaccine strains, based on 20052006 US surveillance data, and that $80 \%$ of influenza isolates identified from the placebo group in Season 2 would be antigenically matched to the vaccine strains, based on the mean and median values of US surveillance results for the preceding 6 years [21]. Based on these estimations and the actual CCI attack rate identified in Season 1, the enrollment target for Season 2 was increased from 3187 to 3900 participants.

\section{Statistical analysis}

The primary analysis was performed on the Per-Protocol (PP) set including participants who received the treatment to which they were randomized, responded to $\geq 1$ post-vaccination active surveillance telephone call, and with no major protocol deviations considered to affect the efficacy or immunogenicity data. Exclusions from the PP set were determined and documented before unblinding. The possible introduction of biases by use of the PP set was assessed by repeating efficacy analyses in the ITI set, which was the PP set plus participants with protocol deviations and treatment errors, and analyzed as randomized. Immunogenicity was evaluated in a randomly selected subset of participants (immunogenicity set).

The primary efficacy analysis was based on the average efficacy over Seasons 1 and 2 and assessed the null hypothesis that the average efficacy in the actively immunized group was $\leq 35 \%$, against the alternative hypothesis that average efficacy was $>35 \%$. Average efficacy was defined as $1-v\left(R_{1} R_{2}\right) \times 100$ where $R_{1}$ and $R_{2}$ were the relative risks of a given disease endpoint in Seasons 1 and 2, respectively. A one-sided $97.5 \% \mathrm{CI}$ was constructed for the average efficacy, and the TIV efficacy target was to be established if the lower bound of the CI was $>35 \%$.

The seroconversion rate was defined as the proportion of participants with a $\geq 4$-fold increase in reciprocal HI titer at Day 21 versus Day 0, or a reciprocal HI titer of $\geq$ 40 from a starting value $<10$; seroprotection rate was the proportion of participants with HI titers $\geq 1: 40$ at Day 21 . The geometric means of reciprocal HI titers (GMTs) were calculated for Day 0 and Day 21 samples, and the geometric mean fold-rises (GMFR; geometric mean of the within-subject fold increases from Day 0 to 21) were also calculated. Lot equivalence was assessed using reciprocal GMTs at Day 21 in the immunogenicity set in Season 1. The Zmin method of Wiens and Iglewicz (WI) was used to assess overall lot equivalence with a two-sided 0.05 significance level and power of $\geq 90 \%$ [22]. One-sided $97.5 \%$ lower confidence bounds (identical to two-sided 95\% lower confidence bounds) on the rate of seroconversion, seroprotection, and GMT were calculated with each Season.
The safety set included participants who received any study treatment and had any post-vaccination safety data. If an incorrect treatment was conclusively documented, participants in the safety set were analyzed based on the treatment they actually received. The incidence of immediate complaints, reactogenicity events from Day 0 to Day 3 , and unsolicited AEs were compared between the TIV and placebo groups using Fisher's exact tests. Any reactogenicity event at grade 2 or 3 with an incidence of $>10 \%$ was assessed using an extended Mantel-Haenszel test. Statistical analyses of safety were performed at the twosided significance level of 0.05 , with no correction for multiple comparisons.

\section{Results}

\section{Participants}

Participant enrollment in Season 1 began 17 September 2005 and follow-up ended 31 May 2006, and enrollment in Season 2 began 16 October 2006 and follow-up ended 6 June 2007. A total of 7611 participants received treatment; 3783 participants received TIV, and 3828 received placebo. A total of 7219 (95\%) of vaccinated participants completed the study (Figure 1). The PP set included 3714 participants in the TIV group, and 3768 participants in the placebo group. No participants with culture-confirmed influenza illness were excluded from the primary analysis.

There were no significant differences between the treatment groups for any baseline characteristic (Table 1). In both groups, $57 \%$ of participants were aged 18-34 years, and $43 \%$ were aged $35-49$ years.

\section{Vaccine efficacy}

The average attack rate for VMCCI over Seasons 1 and 2 was low at $23 / 3714(0.6 \%)$ in the TIV group, and $45 / 3768$ (1.2\%) in the placebo group (Table 2). The VMCCI attack rate in the placebo group in Season $1(1.7 \%)$ was lower than, but similar to, the assumption used for the original sample size estimate (2.0\%). In Season 2, the attack rate for VMCCI in the placebo group was extremely low at $0.7 \%$ and was much lower than the assumed attack rate of VMCCI of $1.6 \%$ that had been used to determine the revised sample size for enrollment in Season 2.

The average efficacy of TIV for the prevention of VMCCI was $46.3 \%$, with a one-sided $97.5 \%$ CI lower bound of $9.8 \%$, thus the null hypothesis of efficacy $\leq 35 \%$ could not be rejected. Attack rates for CCI and LCI over Seasons 1 and 2 were higher than the attack rates of VMCCI, but were still relatively low. In the TIV and placebo groups, attack rates for CCI were $0.8 \%$ and $1.6 \%$, respectively, and the average efficacy of TIV against CCI was $49.3 \%$ with a $97.5 \%$ CI lower bound of $20.3 \%$. The attack rates for LCI in the TIV and placebo groups were $1.2 \%$ and $3.2 \%$, respectively, and the average efficacy of 


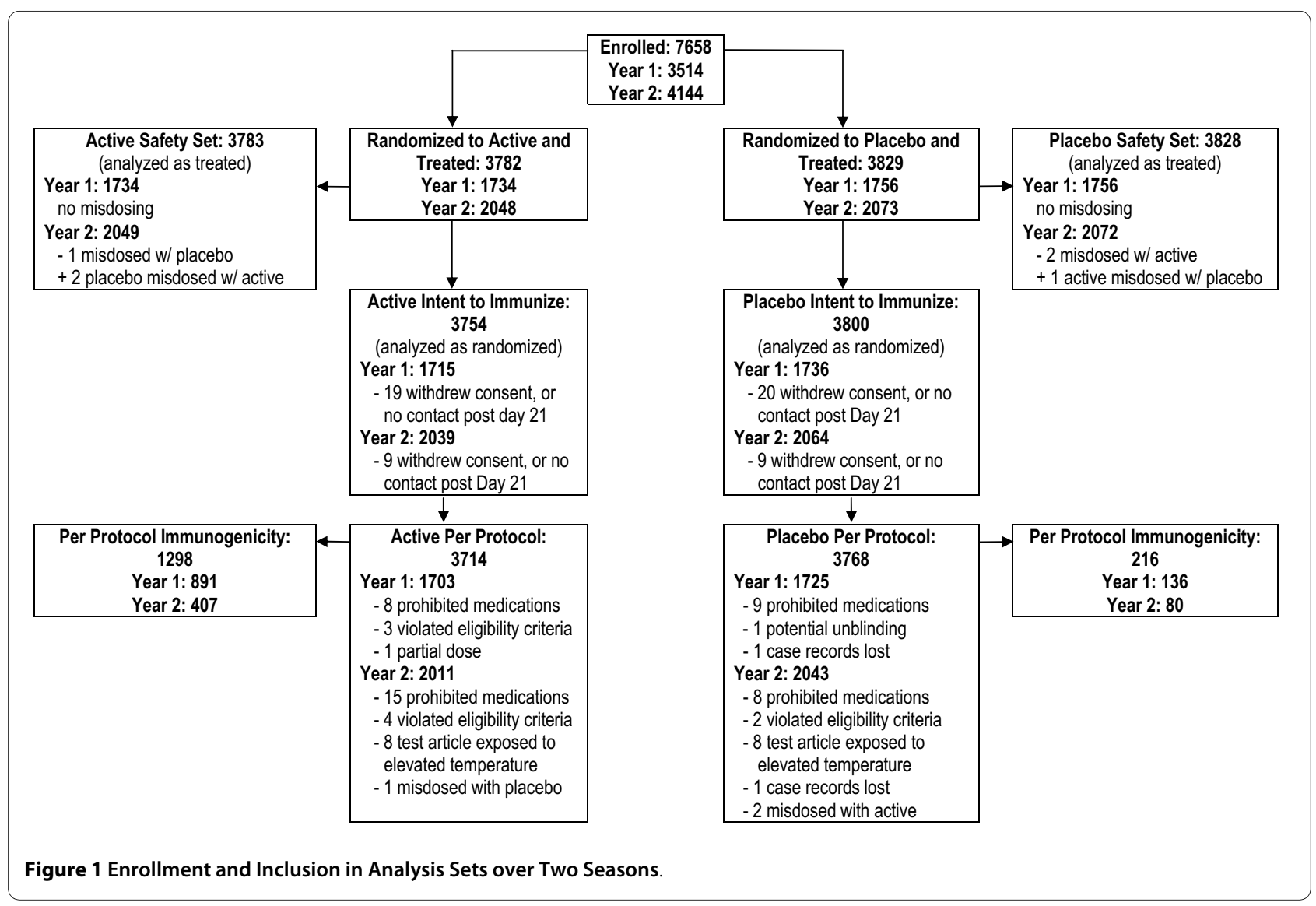

TIV against LCI was $63.2 \%$ with a $97.5 \%$ CI lower bound of $48.2 \%$ (Table 2). The results of the efficacy analyses conducted using the ITI set were essentially identical to those using the PP set; differing by only $0.1 \%$ for each of the VMCCI, CCI, and LCI endpoints (data not shown).
The antigenic characteristics of the influenza A and B viruses isolated from study participants showed good general concordance with CDC surveillance data in the same years [23,24]. In both Seasons 1 and 2, there was limited antigenic drift among A/H3N2 strains, and effi-

Table 1: Demographic and clinical characteristics of study participants.

\begin{tabular}{|c|c|c|}
\hline & $\begin{array}{c}\text { Vaccine } \\
\mathbf{N}=\mathbf{3 7 8 3}\end{array}$ & $\begin{array}{c}\text { Placebo } \\
\mathrm{N}=\mathbf{3 8 2 8}\end{array}$ \\
\hline Mean age, years (SD) & $32.7(9.19)$ & $32.7(9.14)$ \\
\hline 1. Male, n (\%) & 1465 (39) & $1520(40)$ \\
\hline \multicolumn{3}{|l|}{ Age stratum, $\mathrm{n}(\%)$} \\
\hline Age $18-34$ & $2153(57)$ & $2181(57)$ \\
\hline Age $35-49$ & $1630(43)$ & $1647(43)$ \\
\hline \multicolumn{3}{|l|}{ Race, $\mathrm{n}(\%)$} \\
\hline Caucasian & $3166(84)$ & $3237(85)$ \\
\hline Black or African American & $384(10)$ & $362(9)$ \\
\hline Asian & $61(2)$ & $75(2)$ \\
\hline Native Hawaiian or Pacific Islander & $10(<1)$ & $15(<1)$ \\
\hline Native American/Alaskan Native & $18(<1)$ & $9(<1)$ \\
\hline Other & $144(4)$ & $129(3)$ \\
\hline $\begin{array}{l}\text { Received Prior Vaccination ( } \leq 2 \text { years), } \mathrm{n} \\
(\%)\end{array}$ & $727(19)$ & 727 (19) \\
\hline
\end{tabular}


Table 2: Vaccine efficacy against VMCCI, CCI, and LCl in the Per-Protocol analyses.

\begin{tabular}{|c|c|c|c|c|c|c|c|}
\hline & \multicolumn{2}{|c|}{$\begin{array}{c}\text { Season } 1 \\
2005-2006\end{array}$} & \multicolumn{2}{|c|}{$\begin{array}{c}\text { Season } 2 \\
2006-2007\end{array}$} & \multicolumn{2}{|c|}{ Overall } & \multirow{2}{*}{$\begin{array}{c}\text { Overall } \\
\text { efficacy } \\
\text { (97.5\% Cl } \\
\text { lower } \\
\text { bound) }\end{array}$} \\
\hline & $\begin{array}{c}\text { Vaccine } \\
\mathbf{N}=1706 \\
n(\%)\end{array}$ & $\begin{array}{c}\text { Placebo } \\
\text { N= 1725 } \\
\text { n (\%) }\end{array}$ & $\begin{array}{c}\text { Vaccine } \\
\mathbf{N}=\mathbf{2 0 1 1} \\
\mathrm{n}(\%)\end{array}$ & $\begin{array}{c}\text { Placebo } \\
\text { N = 2043 } \\
\text { n (\%) }\end{array}$ & $\begin{array}{c}\text { Vaccine } \\
N=\mathbf{3 7 1 4} \\
n(\%)\end{array}$ & $\begin{array}{c}\text { Placebo } \\
\mathbf{N}=\mathbf{3 7 6 8} \\
\mathbf{n}(\%)\end{array}$ & \\
\hline VMCCI & $14(0.8)$ & $30(1.7)$ & $9(0.4)$ & $15(0.7)$ & $23(0.6)$ & $45(1.2)$ & $\begin{array}{l}46.3 \% \\
(9.8 \%)\end{array}$ \\
\hline $\mathrm{CCl}$ & $19(1.1)$ & $38(2.2)$ & $11(0.5)$ & $22(1.1)$ & $30(0.8)$ & $60(1.6)$ & $\begin{array}{c}49.4 \% \\
(20.3 \%)\end{array}$ \\
\hline LCI & $24(1.4)$ & $69(4.0)$ & $20(1.0)$ & $53(2.6)$ & $44(1.2)$ & $122(3.2)$ & $\begin{array}{c}63.2 \% \\
(48.2 \%)\end{array}$ \\
\hline
\end{tabular}

$\mathrm{VMCCl}$, culture-confirmed influenza $\mathrm{A}$ and $\mathrm{B}$ matching vaccine; $\mathrm{CCl}$, any culture-confirmed influenza $\mathrm{A}$ and $\mathrm{B}$; $\mathrm{LCl}$, any culture- or serologyconfirmed influenza A or $\mathrm{B} ; \mathrm{Cl}$, Confidence Interval

cacy against disease associated with $\mathrm{H} 3 \mathrm{~N} 2$ isolates differed minimally for vaccine-matched strains (VMCCI) and all strains (CCI), or between seasons. No A/H1N1 viruses were isolated in Season 1, and in Season 2, overall efficacy against $\mathrm{A} / \mathrm{H} 1 \mathrm{~N} 1$ was low at $23.9 \%$, although this was based on a very small number of cases (6 in the TIV group, 8 in the placebo group). In Season 1, in which a Yamagata-lineage B virus was used in the vaccine and all $B$ virus isolates were found to be of the Victoria lineage, there was thus by definition no VMCCI, and observed efficacy against $\mathrm{CCI}$ for Victoria lineage viruses was 0 . In Season 2, in which B/Malaysia/2506/04 (a Victoria-lineage virus) was used in the vaccine, the point estimate for efficacy against VMCCI due to influenza B viruses was $100 \%$, while the point estimate for efficacy against disease associated with Yamagata-lineage B viruses was 66\% (estimates again based on small case numbers, with broad 95\% confidence bounds).

The average efficacy of TIV against VMCCI was slightly higher in the 18-34 years stratum at 51.6\% (97.5\% CI lower bound $44.5 \%$ ), than in the $35-49$ years stratum at 44.5\% (97.5\% CI lower bound $-11.8 \%$ ), but this difference was not statistically significant $(\mathrm{p}=0.79)$. Unexpectedly, the average efficacy against VMCCI was much lower in women, $19.4 \%$ (97.5\% CI lower bound $-42.8 \%$ ), than in men, $89.0 \%$ (97.5\% CI lower bound 53.0\%; p value for the male:female contrast 0.0123 ). A similar, although slightly less marked trend between sexes was observed for the average efficacy of CCI and LCI (data not shown). Overall, women in this trial were marginally older than men (mean \pm SD: women $33.3 \pm 9.14$ years; men $31.7 \pm 9.12$ years) and had a slightly higher rate than men of recent (within $\leq 2$ years) previous influenza vaccine (women, $21 \%$; men, $16 \% ; p<0.0001$ ); these differences were similar in the TIV and placebo groups.

In the PP set, ILI symptoms were reported by a total of $362 / 3714$ (10\%) participants in the TIV group, and 427/ 3768 (11\%) participants in the placebo group. An ad hoc analysis was conducted to assess efficacy when the definition of ILI was restricted to include fever (defined by selfobserved oral temperature $\geq 38^{\circ} \mathrm{C}$ ). With this modification, the average efficacy of TIV against VMCCI was $58.0 \%$, with a $97.5 \%$ CI lower bound of $-21.5 \%$ (due to fewer cases), and the average efficacy of TIV against CCI was $66.8 \%$, with a $97.5 \%$ CI lower bound of $7.2 \%$.

\section{Immunogenicity}

The PP immunogenicity set included 1514 randomly selected participants, of which 1298 received TIV and 216 received placebo. TIV was highly immunogenic in Seasons 1 and 2 (Table 3). Immune responses were similar in both seasons for the A/H1N1 and the B virus components. HI GMTs increased 9.94-10.96-fold after immunization for the A/H1N1 component (A/NewCaledonia/20/99 in both seasons), with $68 \%$ seroconversion rates in each season. GMTs increased 11.45-12.36-fold for the B virus components, with $82 \%$ seroconversion to B/Jiangsu/10/03 (a Yamagata lineage virus) in Season 1 and 74\% seroconversion to B/Malaysia/2506/04 (a Victoria lineage virus) in Season 2. Responses differed between seasons for the $\mathrm{A} / \mathrm{H} 3 \mathrm{~N} 2$ component, with a stronger response in Season 1 to the A/New York/55/2004 antigen 
Table 3: Summary of immunogenicity data in the immunogenicity set.

\begin{tabular}{|c|c|c|c|c|}
\hline & \multicolumn{2}{|c|}{$\begin{array}{c}\text { Season } 1 \\
2005-2006\end{array}$} & \multicolumn{2}{|c|}{$\begin{array}{c}\text { Season } 2 \\
2006-2007\end{array}$} \\
\hline & $\begin{array}{l}\text { Vaccine } \\
\mathbf{N}=\mathbf{8 9 1}\end{array}$ & $\begin{array}{l}\text { Placebo } \\
\mathrm{N}=136\end{array}$ & $\begin{array}{l}\text { Vaccine } \\
\mathbf{N}=\mathbf{4 0 7}\end{array}$ & $\begin{array}{l}\text { Placebo } \\
\mathbf{N}=\mathbf{8 0}\end{array}$ \\
\hline \multicolumn{5}{|l|}{$\begin{array}{l}\text { A/New Caledonia } \\
\text { (H1N1) }\end{array}$} \\
\hline Day 0 GMT $(95 \% \mathrm{Cl})^{\dagger}$ & $35.2(32.0,38.6)$ & $31.5(24.7,40.1)$ & $35.5(30.8,40.9)$ & $34.1(24.8,47.0)$ \\
\hline Day 21 GMT $(95 \% \mathrm{Cl})^{+}$ & $385.4(353.0,420.8)$ & $30.9(24.7,38.7)$ & $352.5(309.7,401.3)$ & $38.1(28.4,51.0)$ \\
\hline GMFR $(95 \%$ Cl)‡ & $10.96(9.87,12.18)$ & $0.98(0.75,1.28)$ & $9.94(8.55,11.55)$ & $1.11(0.79,1.56)$ \\
\hline $\begin{array}{l}\text { Seroconversion, } \mathrm{n}(\% ; \\
95 \% \mathrm{Cl} \text { ) }\end{array}$ & $605(68 \% ; 65.0,71.0)$ & $0(0 \%, 00.0,00.0)$ & $275(68 \% ; 63.0,72.0)$ & $1(1 \% ; 00.0,4.0)$ \\
\hline $\begin{array}{l}\text { Day 0 Hl titers } \geq 1: 40, \mathrm{n} \\
(\% ; 95 \% \mathrm{Cl}) \text { ฯ }\end{array}$ & $454(51 \% ; 48.0,54.0)$ & $70(51 \% ; 43.0,60.0)$ & $200(49 \% ; 44.0,54.0)$ & $40(50 \% ; 39.0,61.0)$ \\
\hline $\begin{array}{l}\text { Day } 21 \mathrm{Hl} \text { titers } \geq 1: 40 \text {, } \\
\text { n (\%; } 95 \% \mathrm{Cl}) \text { q }\end{array}$ & $863(97 \% ; 96.0,98.0)$ & $71(52 \% ; 44.0,61.0)$ & 399 (98\%; 97.0, 99.0) & $41(51 \% ; 40.0,62.0)$ \\
\hline \multicolumn{5}{|l|}{ A/New York (H3N2) } \\
\hline Day 0 GMT (95\% Cl) & $16.3(15.2,17.5)$ & $16.2(13.4,19.5)$ & - & - \\
\hline Day 21 GMT $(95 \%$ Cl)† & $258.4(237.0,281.7)$ & $16.5(13.2,20.5)$ & - & - \\
\hline GMFR $(95 \%$ Cl) $\neq$ & $15.84(14.56,17.23)$ & $1.02(0.82,1.26)$ & - & - \\
\hline $\begin{array}{l}\text { Seroconversion, n (\%; } \\
95 \% \mathrm{Cl}) \text { ๆ }\end{array}$ & $756(85 \% ; 82.0,87.0)$ & $1(1)(1 . \% ; 0.0,2.0)$ & & \\
\hline $\begin{array}{l}\text { Day } 0 \mathrm{HI} \text { titers } \geq 1: 40, \mathrm{n} \\
(\% ; 95 \% \mathrm{Cl})\end{array}$ & $273(31 \% ; 28.0,34.0)$ & $41(30 \% ; 22.0,38.0)$ & - & - \\
\hline $\begin{array}{l}\text { Day } 21 \mathrm{HI} \text { titers } \geq 1: 40 \text {, } \\
\mathrm{n}(\% ; 95 \% \mathrm{Cl})\end{array}$ & 837 (94\%; 92.0, 96.0) & $42(31 \% ; 23.0,39.0)$ & - & - \\
\hline \multicolumn{5}{|l|}{$A /$ Wisconsin (H3N2) } \\
\hline Day 0 GMT (95\% Cl) & - & - & $14.9(13.3,16.7)$ & $14.2(11.0,18.4)$ \\
\hline Day 21 GMT $(95 \%$ Cl)† & - & - & $157.6(140.3,177.1)$ & $14.7(11.3,19.1)$ \\
\hline GMFR $(95 \%$ Cl)‡ & - & - & $10.59(9.26,12.11)$ & $1.04(0.77,1.40)$ \\
\hline $\begin{array}{l}\text { Seroconversion, n (\%; } \\
95 \% \mathrm{Cl}) \text { ฯ }\end{array}$ & - & - & $292(72 \% ; 67.0,76.0)$ & $1(1 \% ; 0.0,4.0)$ \\
\hline $\begin{array}{l}\text { Day } 0 \mathrm{HI} \text { titers } \geq 1: 40, \mathrm{n} \\
(95 \% \mathrm{Cl})\end{array}$ & - & - & $116(29 \% ; 24.0,33.0)$ & $23(29 \% ; 19.0,0.39)$ \\
\hline $\begin{array}{l}\text { Day } 21 \mathrm{Hl} \text { titers } \geq 1: 40 \\
(95 \% \mathrm{Cl})\end{array}$ & - & - & $375(92 \% ; 90.0,95.0)$ & $23(29 \% ; 19.0,39.0)$ \\
\hline
\end{tabular}

\section{B/Jiangsu \\ (Yamagata)}

Day 0 GMT $(95 \% \mathrm{Cl}) \dagger$

Day 21 GMT $(95 \% \mathrm{Cl}) \dagger$

$\operatorname{GMFR}(95 \% \mathrm{Cl}) \neq$

Seroconversion, $\mathrm{n}(\%$; $95 \% \mathrm{Cl})$ ๆ

Day $0 \mathrm{HI}$ titers $\geq 1: 40, \mathrm{n}$ $(\% ; 95 \% \mathrm{Cl})$

$\begin{array}{cc}25.4(23.4,27.4) & 23.9(19.6,29.3) \\ 313.5(290.1,338.7) & 24.5(20.1,29.9) \\ 12.36(11.35,13.46) & 1.02(0.82,1.27) \\ 727(82 \% ; 79.0,84.0) & 1(1 \% ; 0.0,2.0) \\ 416(47 \% ; 43.0,50.0) & 63(46 \% ; 38.0,55.0)\end{array}$


Table 3: Summary of immunogenicity data in the immunogenicity set. (Continued)

\begin{tabular}{|c|c|c|c|c|}
\hline $\begin{array}{l}\text { Day } 21 \text { HI titers } \geq 1: 40 \text {, } \\
\text { n }(\% ; 95 \% \mathrm{Cl})\end{array}$ & $873(98 \% ; 97.0,99.0)$ & $65(48 \% ; 39.0,56.0)$ & & \\
\hline Day 0 GMT $(95 \% \mathrm{Cl}) \dagger$ & & & $23.9(19.6,29.3)$ & $35.7(27.2,46.8)$ \\
\hline Day 21 GMT (95\% Cl)† & & & $294.5(263.9,328.7)$ & $38.4(30.0,49.2)$ \\
\hline $\begin{array}{l}\text { Seroconversion, n (\%; } \\
95 \% \mathrm{Cl}) \text { ף }\end{array}$ & - & - & 301 (74\%; 70.0, 78.0) & $1(1 \% ; 0.0,4.0)$ \\
\hline GMFR $(95 \% \mathrm{Cl}) \neq$ & - & - & $11.45(9.98,13.13)$ & $1.08(0.79,1.47)$ \\
\hline $\begin{array}{l}\text { Day } 0 \mathrm{HI} \text { titers } \geq 1: 40, \mathrm{n} \\
(\% ; 95 \% \mathrm{Cl}) \uparrow\end{array}$ & - & - & 179 (44\%; 39.0, 49.0) & $45(56 \% ; 45.0,97.0)$ \\
\hline $\begin{array}{l}\text { Day } 21 \mathrm{HI} \text { titers } \geq 1: 40 \text {, } \\
\text { n }(\% ; 95 \% \mathrm{Cl}) \uparrow\end{array}$ & - & - & $396(97 \% ; 96.0,99.0)$ & $48(60 \% ; 49.0,71.0)$ \\
\hline
\end{tabular}

15.84 fold-rise in GMT and $85 \%$ seroconversion rate) than in Season 2 to the A/Wisconsin/67/2005 antigen (10.59 fold-rise in GMT and 72\% seroconversion rate). Despite this difference, the two-sided 95\% CI lower bound for the proportions of active vaccine recipients attaining post-immunization HI titers $\geq 1: 40$ were $\geq 90 \%$ for all vaccine strains in both seasons.

Treatment with placebo had a negligible effect on $\mathrm{HI}$ titers in both seasons. In view of the unexplained gender difference in efficacy noted above, a post-hoc examination of immune response by gender was performed. The seroconversion rate for the A/New Caledonia H1N1 antigen was lower among women than men in both seasons, and lower GMTs for this virus were found among women (318.6) compared with men (414.6) in Season 2; however, the proportion of men and women with A/New Caledonia/20/99 HI titers $\geq 1: 40$ was $\geq 96 \%$ in both seasons, and there were no other immunogenicity differences of note between men and women (Table 4).

In Season 1, immunogenic lot consistency based on GMT values was demonstrated using the $Z_{\min }$ statistic by the method of Wiens and Iglewciz [22], and according to the study protocol, was not retested in Season 2 (data not shown).

\section{Reactogenicity and safety}

One or more solicited events after immunization occurred in $2487 / 3783$ (66\%) participants in the TIV group and $1675 / 3828(44 \%)$ participants in the placebo group ( $\mathrm{p}<0.0001$ ) (Table 5 ). Common reactogenicity complaints (30 minutes post-vaccination through Day 3) that were significantly more frequent in the TIV versus the placebo group included pain/soreness at the injection site $(51 \%$ and $14 \%$, respectively), tiredness (20\% and $18 \%$, respectively), and myalgia and/or arthralgia (18\% and $10 \%$, respectively). Less frequent, but also significantly associated with active treatment, were malaise and redness and/or swelling at the injection site. Oral temperatures of $\geq 37.5^{\circ} \mathrm{C} / 99.9^{\circ} \mathrm{F}$ were reported by $3 \%$ of TIV recipients and by $1 \%$ of placebo recipients $(\mathrm{p}=0.0005)$; however, the great majority of these reports included maximal temperatures of $\leq 38.5^{\circ} \mathrm{C} / 101.3^{\circ} \mathrm{F}$. From Day 0 through Day 3 post-vaccination, there was no significant association between TIV vaccination and reports of headache, red eyes, cough, sore throat, and hoarseness or pain on swallowing.

In both groups, the most frequent unsolicited AEs from Days 0 through Day 21 with TIV and placebo were pharyngolaryngeal pain $(120 / 3783$ and $120 / 3828$, respectively [both $3 \%$ ]), headache (118/3783 and $121 / 3828$, respectively [both $3 \%$ ]), and fatigue (108/3783 and 120/3828 [both 3\%]). Overall, the rate of AE reports in the TIV group was $808 / 3783(21 \%)$ and in the placebo group was $736 / 3828(19 \%)(\mathrm{p}=0.021)$.

From Day 0 to Final Visit, the incidence of unsolicited AEs by SOC reported by $\geq 1 \%$ of participants was higher with TIV than placebo, with injection site pain $(95 / 3785$ [3\%] and 30/3828 [1\%], respectively; $\mathrm{p}<0.0001)$, and injection site erythema $(45 / 3783[1 \%]$ and $9 / 3828[<1 \%]$, respectively; $\mathrm{p}<0.0001)$ accounting for the betweengroup difference. No other significant differences were seen between groups for incidence rates of AEs by SOC. 
Table 4: Summary of immunogenicity in men and women receiving TIV in the immunogenicity set.

\begin{tabular}{|c|c|c|c|c|}
\hline Year & Antigen & Parameter & $\begin{array}{c}\text { Men } \\
\text { value }(\mathbf{9 5} \% \mathrm{Cl}) \\
\mathbf{N}=\mathbf{4 8 2}\end{array}$ & $\begin{array}{c}\text { Women } \\
\text { value }(95 \% \mathrm{CI}) \\
\mathrm{N}=\mathbf{8 1 6}\end{array}$ \\
\hline Season 1 & $\mathrm{~A} / \mathrm{H} 1 \mathrm{~N} 1$ & \% Seroconversion & $72(67,77)$ & $66(62,70)$ \\
\hline \multirow[t]{8}{*}{$2005-6$} & & Day 21 GMT & $385.9(334.6,445.1)$ & $385.1(344.4,430.7)$ \\
\hline & & Day $21 \% \geq 40$ & $98(96,99)$ & $96(95,98)$ \\
\hline & $\mathrm{A} / \mathrm{H} 3 \mathrm{~N} 2$ & \% Seroconversion & $85(81,89)$ & $85(82,88)$ \\
\hline & & Day 21 GMT & $265.4(231.0,305.0)$ & $254.4(227.7,284.2)$ \\
\hline & & Day $21 \% \geq 40$ & $95(93,97)$ & $93(91,95)$ \\
\hline & B & \% Seroconversion & $83(79,87)$ & $81(77,84)$ \\
\hline & & Day 21 GMT & $329.6(291.3,373.0)$ & $304.6(275.8,336.4)$ \\
\hline & & Day $21 \% \geq 40$ & $99(98,100)$ & $98(96,99)$ \\
\hline Season 2 & $\mathrm{~A} / \mathrm{H} 1 \mathrm{~N} 1$ & \% Seroconversion & $72(65,79)$ & $65(59,71)$ \\
\hline \multirow[t]{8}{*}{$2006-7$} & & Day 21 GMT & $414.2(337.0,508.9)$ & $318.6(269.7,376.3)$ \\
\hline & & Day $21 \% \geq 40$ & $99(97,100)$ & $98(96,99)$ \\
\hline & $\mathrm{A} / \mathrm{H} 3 \mathrm{~N} 2$ & \% Seroconversion & $67(60,74)$ & $75(69,80)$ \\
\hline & & Day 21 GMT & $141.9(117.7,171.1)$ & $168.3(145.1,195.3)$ \\
\hline & & Day $21 \% \geq 40$ & $90(86,95)$ & $93(90,96)$ \\
\hline & B & \% Seroconversion & $73(66,80)$ & $74(69,80)$ \\
\hline & & Day 21 GMT & $290.9(244.8,345.7)$ & $296.8(257.2,342.5)$ \\
\hline & & Day $21 \% \geq 40$ & $97(95,100)$ & $97(95,99)$ \\
\hline
\end{tabular}

GMT, geometric mean titer

Unsolicited SAEs were uncommon, and were reported by $1 \%$ of participants in both treatment groups. Overall, $44 / 3783(1 \%)$ and $39 / 3728(1 \%)$ of participants in the TIV and placebo groups, respectively, experienced at least 1 SAE. All SAE types occurred in $\leq 1 \%$ of participants. None of the SAEs were considered by the investigators to be vaccine-related. One subject in the placebo group had a fatal road traffic accident during the study. Severity of ILI was not systematically assessed; a single subject in the placebo group was hospitalized with a diagnosis of influenza.

\section{Pregnancies}

There were a total of 25 pregnancies in the TIV group, and 32 in the placebo group, of which 4 (16\%: $95 \%$ CI; $5 \%$, $36 \%)$ and 7 (22\%: 95\% CI; 9\%, 40\%), respectively, ended in spontaneous abortion. Three pregnancies were electively terminated. One placebo recipient was induced at 34 weeks because of intrauterine growth retardation, and the infant was reported to have 2 hernias, which were surgically repaired, and a heart murmur. Two pregnant participants were lost to follow up, and there were 18 (72\%) and $22(69 \%)$ full-term births with healthy infants in the TIV and placebo groups, respectively.

\section{Discussion}

Although inactivated influenza vaccines are recommended for many populations, estimates as to their true efficacy vary widely. It is commonly held that the efficacy of TIVs is 60 to $80 \%$ in healthy younger adults and similar in children over the age of 2 years, but less in the elderly (particularly the chronically-ill or institutionalized) [2529]. The degree of antigenic match between the vaccine and circulating virus strains, the exact illness endpoint used, and the surveillance methodology in clinical trials can influence efficacy estimates. Efficacy data concerning children under 2 years of age are very limited.

This placebo-controlled, randomized study was conducted to assess the efficacy, safety, and immunogenicity of a TIV over the 20052006 (Season 1) and 20062007 (Season 2) influenza seasons in healthy adults in the US. The primary endpoint was protection against $\mathrm{VMCCI}$, as 
Table 5: Incidence of TIV reactogenicity events from Day $0^{+}$to Day 3 in the Safety Set

\begin{tabular}{|c|c|c|c|}
\hline Symptom & $\begin{array}{c}\text { TIV } \\
\mathrm{N}=\mathbf{3 7 8 3} \mathrm{n}(\%)\end{array}$ & $\begin{array}{c}\text { Placebo } \\
N=3828 n \text { (\%) }\end{array}$ & P-valueף \\
\hline $\begin{array}{l}\text { At least } 1 \text { vaccine } \\
\text { reactogenicity event }\end{array}$ & $2487(66)$ & $1675(44)$ & $<0.0001$ \\
\hline Fever ${ }^{\ddagger}$ & $96(3)$ & $55(1)$ & 0.0005 \\
\hline Injection site pain/soreness & $1933(51)$ & $530(14)$ & $<0.0001$ \\
\hline Injection site redness & $475(13)$ & $234(6)$ & $<0.0001$ \\
\hline Injection site swelling & $418(11)$ & $109(3)$ & $<0.0001$ \\
\hline Myalgia and/or arthralgia & $692(18)$ & $389(10)$ & $<0.0001$ \\
\hline Headache & $683(18)$ & $716(19)$ & 0.5491 \\
\hline Tiredness & $761(20)$ & $678(18)$ & 0.0049 \\
\hline Chills & $158(4)$ & $136(4)$ & 0.1526 \\
\hline Malaise & $338(9)$ & $236(6)$ & $<0.0001$ \\
\hline Red eyes & $250(7)$ & $231(6)$ & 0.2772 \\
\hline Swelling of the face & $51(1)$ & $37(<1)$ & 0.1327 \\
\hline Cough & $286(8)$ & $250(7)$ & 0.0719 \\
\hline $\begin{array}{l}\text { Chest tightness or difficulty in } \\
\text { breathing }\end{array}$ & $128(3)$ & $107(3)$ & 0.1274 \\
\hline $\begin{array}{l}\text { Sore throat, hoarseness or } \\
\text { pain on swallowing }\end{array}$ & $324(9)$ & $344(9)$ & 0.5689 \\
\hline
\end{tabular}

requested by US regulatory authorities, rather than the frequently cited endpoints of CCI or LCI. Although the LCI endpoint has been questioned by some authors [15], it is still widely used, and constitutes the primary endpoint for the majority of the published TIV efficacy experience.

Despite a good degree of 'vaccine-match' among the influenza isolates in the trial (75.5\%), and a strong immune response to the vaccine in both years, the average efficacy over Seasons 1 and 2 of TIV against VMCCI was only $46.3 \%$, with a one-sided $97.5 \%$ CI lower bound of $9.8 \%$. While the vaccine was clearly efficacious relative to placebo (one-sided $97.5 \%$ CI lower bound for efficacy excluded 0 ), these results did not satisfy the pre-defined criterion for success, i.e. exclusion of efficacy of $\leq 35 \%$ (as evidenced by a lower $97.5 \% \mathrm{CI}$ of $>35 \%$ ). The results were similar for CCI, with an average efficacy of $49.4 \%$, with one-sided $97.5 \%$ CI lower bound of $20.3 \%$, the CI becoming narrower due to the greater number of cases.

Efficacy was higher for the LCI endpoint, at $63.2 \%$, with one-sided $97.5 \%$ CI lower bound of $48.2 \%$; this is generally consistent with previously reported TIV efficacy esti- mates in studies using this endpoint $[12,14,30]$. While an intrinsic bias of the LCI endpoint in favor of the vaccine has been noted $[15,16]$ and, therefore, limits its utility for regulatory purposes, it should also be noted that this endpoint may improve sensitivity relative to culture. In addition, the endpoint of LCI has been used in many prior clinical trials of TIV, yielding estimates of TIV efficacy in adults of about $70 \%[14,15]$. Thus, the estimated vaccine efficacy against LCI in this trial is consistent with that estimated in prior trials of other TIVs.

A key factor influencing efficacy findings in influenza vaccine studies is the disease attack rate, and the unpredictable nature of epidemic intensity poses a challenge when planning influenza vaccine efficacy trials $[12,13,25,31]$. Our study was powered on the assumptions that vaccine efficacy would approximate $70 \%$, based on the prior literature reporting culture-confirmed influenza endpoints, and that the attack rate for CCI in the placebo group would be $2.0 \%$ across both seasons. The attack rate assumption was adjusted in Season 2 to $1.6 \%$ based on the regulatory request to adopt VMCCI as the primary endpoint, and the sample size correspondingly increased. 
The actual VMCCI attack rate in placebo recipients in Season 1 was close to our prediction, but in Season 2 the attack rate was less than half of that predicted, and this had a large negative impact on the precision of the average efficacy estimate. Despite this, it was also clear that the efficacy observed did not differ markedly between the seasons, and might not have sufficed to exclude the 35\% lower bound of the primary hypothesis even if the VMCCI attack rates in Season 2 had matched those in Season 1.

Similar to the findings of our study, the Centers for Disease Control and Prevention surveillance data summaries for the 2005-2006 and 2006-2007 influenza seasons in the US suggest that these were relatively mild influenza seasons $[23,24]$. The weekly percentage of outpatient visits to US sentinel providers for ILI peaked twice in the 20052006 season at $3.3 \%$ and $3.2 \%$ (baseline $2.2 \%$ ), and peaked twice in the 2006-2007 season at 3.0\% and 3.5\% (baseline $2.1 \%)[23,24]$. In the prior three seasons, the peak percentage of outpatient visits for ILI had ranged from $2.3 \%$ to $7.6 \%[23,24]$.

A negative impact of low attack rates in a given season on point estimates of efficacy has been well documented. Previous studies of a TIV product, involving the same investigators, methods, and source populations over successive seasons, have reported dramatic differences in efficacy estimates between seasons, without obvious explanation based on circulating strain match to the vaccine $[12,13]$. In these studies, the low efficacy estimates were found in seasons with lower overall attack rates $[12,13]$; a similar experience has occurred in pediatric trials [31]. While a clear reason for this phenomenon is not apparent, it can be speculated that low background attack rates may result from either relatively high pre-existing population resistance to the circulating strain(s), or modest intrinsic virulence of the circulating strains, such that only intense exposures lead to disease. In either case, the attainable impact of a vaccine might be blunted. Primary detection of influenza virus infections in this study was by means of viral culture; with the use of molecular methods confined to characterization of isolates. Other reports have suggested that the use of RT-PCR as a primary detection tool may improve the rate of ascertainment of influenza virus infections by 20 to $30 \%$ $[12,13,31,32]$, but this maneuver does not appear, in and of itself, to have a major impact on point estimates of efficacy.

As suggested previously, a further factor that may compromise vaccine efficacy is the extent of antigenic drift and the degree of match between vaccine and circulating viruses $[11,12,33]$. In our study, the majority of cases in both seasons were due to influenza A/H3N2 viruses, and antigenic drift was modest and had little apparent impact. However, only 5/17 cases of culture-confirmed influenza
B disease were due to viruses of the same lineage as the vaccine strain in the relevant season (data not shown), and average efficacy against non-matching B strains was low $(\sim 16 \%)$; this result clearly reduced the estimate of efficacy against all CCI.

The definition of influenza-like illness (ILI) in our study required that illness impeded normal daily activities, with cough plus $\geq 1$ other symptom from a panel including both respiratory and systemic complaints, so this allowed for a diagnosis without need for a systemic symptom (i.e. fever, myalgia/arthralgia). Cough has been previously found to be one of the most sensitive clinical indicators of influenza, but has little specificity, whereas fever is a classically-cited characteristic of influenza, with substantial positive predictive value, but lower sensitivity [34]. Fever was not made a requirement for ILI in our study in order to maximize sensitivity. However, our ad hoc analyses showed that narrowing of the case definition to require fever reduced the case rate but notably improved the apparent efficacy of TIV. A potential interpretation of this finding may be that TIV is most effective against severe manifestations of influenza, and less so against illnesses with predominantly respiratory symptoms. Although efficacy against severe disease is desirable, the relatively sensitive but non-specific case definition in our study may have allowed for the inclusion of respiratory illness caused by mixed infections in which influenza viruses may have been minor or coincidental. An additional factor that could have compromised the results is the highly decentralized source of the specimens, and the transportation of specimens to a centralized laboratory may not have been optimal for the recovery of influenza viruses from nasopharyngeal swabs.

An unexpected finding of the study was the difference between men and women for the primary endpoint: men, 89.0\%; and women, $19.4 \%$. Women were slightly older than men (33.3 and 31.7 years, respectively), and were more likely to have had previous recent influenza vaccination ( $21 \%$ and $16 \%$, respectively). These between-gender differences were reflected in both treatment groups, which were well matched for all baseline characteristics. In addition, all three viruses were culture-confirmed in men and women, there was no differential temporal clustering of cases, and serum HI titers did not differ meaningfully between the sexes (data not shown); this is in contrast to a previous large study of a TIV, which reported significantly higher GMT responses in women than men, regardless of age or dose or influenza strain [35]. The attack rate of VMCCI among placebo-treated men and women was similar $(1.27 \%$ and $1.14 \%$, respectively), which suggests that both sexes had a similar level of exposure. At present, the difference in values between men and women remains unexplained and must be viewed with caution because a further subdivision of the 
already-small case numbers results in very broad CIs about the efficacy estimates.

In terms of seroconversion and rates of attainment of post-vaccinal HI reciprocal titers $\geq 40$ ("seroprotection" rates), the TIV in this study fulfilled the immunogenicity criteria for the accelerated approval of seasonal influenza vaccines established by the US FDA Center for Biologics Evaluation and Research for all strains in both seasons [36]. In addition, manufacturing consistency was confirmed for three consecutive TIV lots in the 2005-2006 season, based on GMT values. Vaccine immunogenicity as measured by induction of hemagglutination-inhibiting antibodies provides a marker of potential vaccine effectiveness in the absence of viral circulation and has therefore been, and continues to be, a useful tool for the registration of influenza vaccines. Nevertheless, definitive demonstration of a single antibody titer which constitutes an absolute correlate of protection for all virus strains remains elusive, and the current study was not designed to demonstrate this.

The reactogenicity events reported in the study were consistent with those commonly reported with TIVs. Reactogenicity events that were significant in the TIV versus placebo group included injection site pain, injection site redness and swelling, myalgias, arthralgias, fever and fatigue; the majority of events were mild (grade 1) in severity. There was a slightly higher incidence of spontaneous AEs in the TIV versus placebo group reported up to 21 days post-vaccination, and this was primarily due to the persistence of injection site pain and redness. Overall, the results suggest that the safety profile of TIV was acceptable, and consistent with the historical performance of similar products.

\section{Conclusions}

This randomized, placebo-controlled trial showed that the average efficacy of TIV for the prevention of VMCCI over the 2005-2006 and 2006-2007 influenza seasons was $46.3 \%$. Although the differences between the vaccine and placebo groups in the primary endpoint did not meet the pre-defined criterion for success, the TIV clearly demonstrated clinical benefit (i.e. it was readily differentiated from placebo by all culture and/or laboratory-confirmed influenza endpoints) and the primary endpoint result must be interpreted with some caution in view of the very low influenza attack rates in both seasons; a factor which has previously been associated with low efficacy estimates in influenza vaccine trials. Furthermore, the immune responses to TIV fulfilled the licensure criteria for seasonal influenza vaccines [36]. Overall, TIV had a safety profile that was considered to be acceptable, and was consistent with other inactivated influenza vaccines.

\section{Competing interests}

GSK Biologicals was the funding source and was involved in all stages of the study conduct and analysis. GSK Biologicals also took in charge all costs associated with the development and the publishing of this manuscript. The corresponding author had full access to the data, and final responsibility for submission of the manuscript for publication.

$L J$ has received research funding from manufacturers of influenza vaccines, including GSK, Sanofi Pasteur, and Novartis and has served as a consultant to GSK and Novartis. Dr Harry Keyserling received grant support from GlaxoSmithKline to conduct the study. JB has no conflict of interest to declare. NB is a former employee of GlaxoSmithKline Biologicals and reports ownership of equity or stock options. LFF is a full-time employee of GlaxoSmithKline. JT discloses having received laboratory support from GSK and MerciaPharma, as well as clinical trial support from Protein Sciences Corporation, Vaxinnate, Ligocyte, Wyeth, Bavarian Nordic, Sanofi and PaxVax. JT discloses scientific advisory board activities for Immune Targeting Systems and Toyama Chemical Concern.

\section{Authors' contributions}

All authors participated in the design, implementation, analysis and interpretation of the study. All authors read and approved the final manuscript. LF and NB were involved in all phases of the study, and led the clinical team at GSK Biologicals. LAJ, MJG and HLK led the clinical team at their respective centers, JB conducted the data analysis. JJT managed the team responsible for detailed antigenic characterization and vaccine match analysis.

\section{Acknowledgements}

We are grateful to the National Institute for Biological Standards and Control (NIBSC, UK) for providing the vaccine virus strains. The authors are indebted to the participating clinicians, sub-investigators, nurses, research coordinators, and laboratory technicians at the study site and the sponsor's project staff for their support and contributions throughout the study. We also want to thank the study volunteers. We would like to thank the laboratory staff for their excellent, sedulous immunological laboratory work. Finally we would like to thank Annick Moon (independent, UK) for her assistance in preparing the manuscript and Isabelle Camby for coordination.

\section{Author Details}

1 Group Health Research Institute, Seattle, Washington, USA, 2Section of Pediatric Infectious Diseases, Scott \& White Memorial Hospital and Clinic, Texas A\&M University Health Science Center College of Medicine, Temple, Texas, USA 3 Division of Pediatric Infectious Diseases, Emory University School of Medicine, Atlanta, Georgia, USA, 4 Veristat, Inc, Holliston, Massachusetts, USA, ${ }^{5}$ GlaxoSmithKline Biologicals, Laval, Quebec, Canada, ${ }^{\circ}$ Variation Biotechnologies Inc, Gatineau, Québec, Canada, ${ }^{7}$ GlaxoSmithKline Biologicals, Columbia, Maryland, USA and 8 Infectious Diseases Unit, University of Rochester Medical Center, Rochester, NY, USA

Received: 28 April 2009 Accepted: 17 March 2010 Published: 17 March 2010

\section{References}

1. Schanzer DL, Langley JM, Tam TW: Hospitalization attributable to influenza and other viral respiratory illnesses in Canadian children. Pediatr Infect Dis J 2006, 25(9):795-800.

2. Thompson WW, Shay DK, Weintraub E, Brammer L, Bridges CB, Cox NJ, Fukuda K: Influenza-associated hospitalizations in the United States. JAMA 2004, 292(11):1333-1340.

3. Thompson WW, Shay DK, Weintraub E, Brammer L, Cox N, Anderson LJ, Fukuda K: Mortality associated with influenza and respiratory syncytial virus in the United States. JAMA 2003, 289(2):179-186.

4. Molinari NA, Ortega-Sanchez IR, Messonnier ML, Thompson WW, Wortley PM, Weintraub E, Bridges CB: The annual impact of seasonal influenza in the US: measuring disease burden and costs. Vaccine 2007, 25(27):5086-5096.

5. Thompson WW, Comanor L, Shay DK: Epidemiology of seasonal influenza: use of surveillance data and statistical models to estimate the burden of disease. J Infect Dis 2006, 194(Suppl 2):S82-91.

6. Li S, Leader S: Economic burden and absenteeism from influenza-like illness in healthy households with children (5-17 years) in the US. Respir Med 2007, 101(6):1244-1250 
7. Nichol KL, D'Heilly SJ, Greenberg ME, Ehlinger E: Burden of influenza-like illness and effectiveness of influenza vaccination among working adults aged 50-64 years. Clin Infect Dis 2009, 48(3):292-298.

8. World Health Organization: WHO Global Influenza Surveillance Network. [http://www.who.int/csr/disease/influenza/surveillance/en/ index.html]. [Accessed March 2010]

9. World Health Organization: Recommendations for influenza vaccines. [http://www.who.int/csr/disease/influenza/vaccinerecommendations/ en/index.html]. [Accessed March 2010]

10. Wright P, Webster R: Orthomyxoviruses. In Fields virology 4th edition. Edited by: Knipe DM, Howley PM, Griffin DE, et al. Philadelphia, PA: Lippincott Williams \& Wilkins; 2001:1534-1579.

11. Nichol KL, Mendelman PM, Mallon KP, Jackson LA, Gorse GJ, Belshe RB, Glezen WP, Wittes J: Effectiveness of live, attenuated intranasal influenza virus vaccine in healthy, working adults: a randomized controlled trial. JAMA 1999, 282(2):137-144

12. Ohmit SE, Victor JC, Rotthoff JR, Teich ER, Truscon RK, Baum LL, Rangarajan B, Newton DW, Boulton ML, Monto AS: Prevention of antigenically drifted influenza by inactivated and live attenuated vaccines. N Eng/ $\mathrm{J}$ Med 2006, 355(24):2513-2522

13. Ohmit SE, Victor JC, Teich ER, Truscon RK, Rotthoff JR, Newton DW Campbell SA, Boulton ML, Monto AS: Prevention of symptomatic seasonal influenza in 2005-2006 by inactivated and live attenuated vaccines. J Infect Dis 2008, 198(3):312-317.

14. Jefferson TO, Rivetti D, Di Pietrantonj C, Rivetti A, Demicheli V: Vaccines for preventing influenza in healthy adults. Cochrane Database Syst Rev 2007:CD001269.

15. Edwards KM, Dupont WD, Westrich MK, Plummer WD Jr, Palmer PS, Wright PF: A randomized controlled trial of cold-adapted and inactivated vaccines for the prevention of influenza A disease. J Infect Dis 1994, 169(1):68-76.

16. Treanor JJ, Kotloff K, Betts RF, Belshe R, Newman F, lacuzio D, Wittes J, Bryant M: Evaluation of trivalent, live, cold-adapted (CAIV-T) and inactivated (TIV) influenza vaccines in prevention of virus infection and illness following challenge of adults with wild-type influenza A (H1N1), A (H3N2), and B viruses. Vaccine 1999, 18(9-10):899-906

17. US Food and Drug Administration: Product Approval Information Licensing Action. 2006 [http://www.fda.gov/BiologicsBloodVaccines/ Vaccines/ApprovedProducts/ucm112909.htm]. Accessed March 2010

18. Centers for Disease Control: Prevention and Control of Influenza. Recommendations of the Advisory Committee on Immunization Practices (ACIP). [http://www.cdc.gov/mmwr/preview/mmwrhtml/ rr5408a1.htm]. Accessed March 2010

19. Redlberger M, Aberle S, Heinz F, Popow-Kraupp T: Dynamics of antigenic and genetic changes in the hemagglutinins of influenza $\mathrm{A} / \mathrm{H} 3 \mathrm{~N} 2$ viruses in three consecutive seasons (2002/2003 to 2004/2005) in Austria. Vaccine 2007, 25:6061-6069.

20. Kendal A, Pereira M, Skehel J: Hemagglutination inhibition. Concepts and procedures for laboratory-based influenza surveillance Edited by: Kendal AP, Pereira MS, Skehel JJ. Atlanta: Centers for Disease Control and Prevention and Pan-American Health Organization; 1982:B17-35.

21. Centers for Disease Control: Flu activity and surveillance. [http:// www.cdc.gov/flu/weekly/fluactivity.htm]. Accessed March 2010

22. Wiens B, Iglewicz B: Design and analysis of three treatment equivalence trials. Control Clin Trials 2001, 21:127-137.

23. Centers for Disease Control: 2005-2006 influenza season summary. [http://www.cdc.gov/flu/weekly/weeklyarchives2005-2006/0506summary.htm]. Accessed March 2010

24. Centers for Disease Control: 2006-2007 influenza season summary. [http://www.cdc.gov/flu/weekly/weeklyarchives2006-2007/0607summary.htm]. [Accessed March 2010]

25. Beran J, Wertzova V, Honegr K, Kaliskova E, Havlickova M, Havlik J, Jirincova $H$, Van Belle P, Jain V, Innis B, et al:: Challenge of conducting a placebocontrolled randomized efficacy study for influenza vaccine in a season with low attack rate and a mismatched vaccine $B$ strain: a concrete example. BMC Infect Dis 2009, 9:2

26. Jefferson T, Rivetti A, Harnden A, Di Pietrantonj C, Demicheli V: Vaccines for preventing influenza in healthy children. Cochrane Database Syst Rev 2008, 16(2):CD004879

27. Langley J, Faughnan M: Prevention of influenza in the general population. CMAJ 2004, 171:1213-1222.
28. Rudenko LG, Arden NH, Grigorieva E, Naychin A, Rekstin A, Klimov Al Donina S, Desheva J, Holman RC, DeGuzman A, et al.: Immunogenicity and efficacy of Russian live attenuated and US inactivated influenza vaccines used alone and in combination in nursing home residents. Vaccine 2000, 19(2-3):308-318

29. Zangwill KM, Belshe RB: Safety and efficacy of trivalent inactivated influenza vaccine in young children: a summary for the new era of routine vaccination. Pediatr Infect Dis J 2004, 23(3):189-197.

30. Langley J, Faughnan M: Prevention of influenza in the general population. CMAJ 2004, 171:1213-1222

31. Hoberman A, Greenberg DP, Paradise JL, Rockette HE, Lave JR, Kearney DH, Colborn DK, Kurs-Lasky M, Haralam MA, Byers CJ, et al:: Effectiveness of inactivated influenza vaccine in preventing acute otitis media in young children: a randomized controlled trial. JAMA 2003, 290(12):1608-1616.

32. Zambon M, Hays J, Webster A, Newman R, Keene O: Diagnosis of influenza in the community: relationship of clinical diagnosis to confirmed virological, serologic, or molecular detection of influenza. Arch Intern Med 2001, 161(17):2116-2122.

33. Belshe RB, Gruber WC, Mendelman PM, Cho I, Reisinger K, Block SL, Wittes J, lacuzio D, Piedra P, Treanor J, et al.: Efficacy of vaccination with live attenuated, cold-adapted, trivalent, intranasal influenza virus vaccine against a variant (A/Sydney) not contained in the vaccine. J Pediatr 2000, 136(2):168-175

34. Nichol KL: Heterogeneity of influenza case definitions and implications for interpreting and comparing study results. Vaccine 2006, 24(4446):6726-6728.

35. Engler RJ, Nelson MR, Klote MM, VanRaden MJ, Huang CY, Cox NJ, Klimov A, Keitel WA, Nichol KL, Carr WW, et al:: Half- vs full-dose trivalent inactivated influenza vaccine (2004-2005): age, dose, and sex effects on immune responses. Arch Intern Med 2008, 168(22):2405-2414.

36. US Food and Drug Administration: Guidance for Industry. Clinical Data Needed to Support the Licensure of Seasonal Inactivated Influenza Vaccines. [http://www.fda.gov/cber/guidelines.htm]. Accessed March 2010

\section{Pre-publication history}

The pre-publication history for this paper can be accessed here: http://www.biomedcentral.com/1471-2334/10/71/prepub

doi: 10.1186/1471-2334-10-71

Cite this article as: Jackson et al., Safety, efficacy, and immunogenicity of an inactivated influenza vaccine in healthy adults: a randomized, placebo-controlled trial over two influenza seasons BMC Infectious Diseases 2010, 10:7

\section{Submit your next manuscript to BioMed Centra and take full advantage of:}

- Convenient online submission

- Thorough peer review

- No space constraints or color figure charges

- Immediate publication on acceptance

- Inclusion in PubMed, CAS, Scopus and Google Scholar

- Research which is freely available for redistribution 